\title{
LA EDUCACIÓN MEDIÁTICA EN LAS TITULACIONES DE EDUCACIÓN Y COMUNICACIÓN DE LAS UNIVERSIDADES ESPAÑOLAS. ANÁLISIS DE LOS RECURSOS RECOMENDADOS EN LAS GUÍAS DOCENTES
}

Carmen Marta-Lazoํㅜㄹ Universidad de Zaragoza. España. cmarta@unizar.es

María del Mar Grandío Pérez: Universidad de Murcia. España. mgrandio@um.es

José Antonio Gabelas Barroso: Universidad de Zaragoza. España. jgabelas@unizar.es

\section{RESUMEN}

La educación mediática es una asignatura que se integra de manera reciente, como materia optativa, en los planes de estudio de algunas de las titulaciones de Comunicación y Educación. Este artículo tiene como objetivo analizar la modalidad de los recursos que se utilizan como fuentes básicas y complementarias en las guías docentes de las asignaturas directamente relacionadas con la Educación Mediática en la totalidad de planes de estudio vigentes en Educación y Comunicación en el territorio español. Esta investigación se incluye dentro del Proyecto de Investigación I+D “La enseñanza universitaria ante la competencia en comunicación audiovisual en un entorno digital" (EDU2010-21395-C03-01). A través de un análisis de contenido cuantitativo de las guías, este estudio delimita aspectos como el tipo de soporte que predomina, el año de edición y la procedencia. Así mismo desarrolla una comparativa entre los recursos integrados en guías de titulaciones de Educación y en guías docentes de estudios de Comunicación. Advertimos la antigüedad de los recursos y la mínima aparición de fuentes internacionales o producidas en otros idiomas que, obviamente, delimitan la enseñanza de esta materia en las universidades españolas.

PALABRAS CLAVE: Educación mediática - Comunicación - Recursos recomendados - Guías Docentes.

\footnotetext{
1 Carmen Marta-Lazo: Profesora Titular de Comunicación Audiovisual y Publicidad de la Universidad de Zaragoza, España. Investigadora Principal de Grupo de Investigación en Comunicación e Información Digital (GICID).

Correo: cmarta@unizar.es
} 


\title{
MEDIA LITERACY WITHIN COMMUNICATION AND EDUCATION DEGREES IN THE SPANISH HIGHER EDUCATION. ANALYSIS OF RECOMMENDED RESOURCES IN THE TEACHING GUIDES
}

\begin{abstract}
Media literacy has been recently integrated as an optional course within the Communciation and Education Degrees in Spanish Higher Education. This article aims to analyze the recommended material in the total of teaching guides in the courses related to media education in these bachelor degrees. This study comes from the Research Project "Audiovisual communication skills in the digital environment. A diagnosis of the requirement in three social settings" (EDU2010-21395-C03-01). Applying a quantative approach to the total of teaching guides objet of study, this article explores the most used type of material by teachers, date of edition and place of procedence. A comparation between resources of the Comunication degree and the Education degree is also presented. As conclusions, it will be underlined how books and other print material are mainly recommended in the teaching guides, but websites and audiovisual sources are hardly recommended. Besides, bibliography is not recently updated and the international references are barly mentioned.
\end{abstract}

KEYWORDS: Media literacy - Communication - Education - Recommended resources - teaching guides.

\section{INTRODUCCIÓN}

La Educación Mediática, bautizada también con otros conceptos dependiendo del país en el que se aborde, tales como "Educomunicación", "Educación para los medios" o "Media Literacy", es la disciplina que aborda los territorios comunes en los que se da cita la comunicación y la educación de manera híbrida y convergente, con el fin de trabajar la educación para los medios y los medios para la educación.

La importante convergencia y sinergia entre los campos de la comunicación y de la educación ya se constató en la Declaración sobre la enseñanza de los medios, promulgada en el Simposium Internacional, celebrado en 1982 en Grunwal (Alemania), donde se concluyó que en la postmodernidad es necesaria la unión entre ambas disciplinas. Entre las funciones que se atribuyeron a los medios, destaca que deben servir como canales en 'la participación de los ciudadanos en la sociedad' y las funciones de los sistemas políticos y educativos deben pasar por 'promover entre los ciudadanos una compresión crítica de los fenómenos de la comunicación' (Marta-Lazo y Grandío Pérez, 2012: 141). De este modo, la Comisión Europea (2007: 1-9) definió Media literacy como habilidad de acceder a los medios, entender y evaluar de forma crítica diferentes aspectos de los medios y del contenido mediático, así como crear mensajes comunicativos en una variedad de contextos. 
En el plano científico, los resultados de las investigaciones empíricas del ámbito académico ofrecen datos constantes sobre el estado de la educación mediática de los ciudadanos, al mismo tiempo que proponen futuras líneas de actuación a estos agentes sociales tanto en el ámbito político como educativo. En este contexto, las ambiciones teóricas, políticas y pedagógicas en España en el ámbito de la educación mediática son grandes, ya que se consideran prioritarias y necesarias para el empoderamiento (empowerment) y educación crítica (critical literacy) del ciudadano (Marta-Lazo y Grandío Pérez, 2013: 115). Se estima que la alfabetización audiovisual y multimedia del profesorado es una asignatura pendiente, previa a la propia alfabetización de los jóvenes, que necesita con carácter de urgencia un tiempo, un espacio y un contenido curricular (Gabelas, 2007: 71). Entre otras investigaciones, destaca el proyecto dirigido por Ferrés Prats titulado "Competencia Mediática. Investigación sobre el grado de competencia de la ciudadanía en España" (2011) que puso de manifiesto el elevado porcentaje de incompetencia audiovisual que poseen la mayoría de los ciudadanos en todas las dimensiones competenciales analizadas: Lenguaje; Tecnología; Procesos de producción y programación; Ideología y valores; Estética; y Recepción y audiencia (Marta-Lazo y Grandío-Pérez, 2013: 115-130) y que destaca la necesidad de incorporar materias y acciones de educación mediática entre los ciudadanos españoles.

En este contexto, se hace relevante que, desde las instituciones académicas y en todos los niveles educativos, desde la educación infantil y primaria, hasta la secundaria y universitaria, se integre el aprendizaje de la Educación Mediática para conseguir desarrollar el sentido crítico y creativo para saber analizar y producir los mensajes en la sociedad actual. En concreto, "la Universidad no debe alejarse del contexto social actual. En la Sociedad del Conocimiento en la que habitamos, es fundamental enseñar a pensar para la vida $y$, en esa realidad, las tecnologías digitales han adquirido una dimensión tan vital como que estar 'alfabetizado digitalmente' es toda una necesidad" (Osuna Acedo, Marta-Lazo y Aparici Merino, 2013).

En la actualidad, aunque la educación mediática se considera fundamental para el pleno desarrollo de los ciudadanos de la sociedad contemporánea, no se encuentra potenciada de manera suficiente en los planes de estudio a nivel escolar ni universitario (Aguaded, 2011: 7). Los escenarios virtuales redimensionan el ámbito sociológico en el que se desenvuelven las diversas culturas juveniles y adultas, con las redes sociales como un factor socializador de primer orden (Gabelas y Marta-Lazo, 2011: 10). Los nuevos entornos digitales y la nueva configuración y despliegue de nuevas aplicaciones y redes sociales plantean una mayor necesidad, si cabe, de fomentar el desarrollo de las competencias citadas. De hecho, como ya hemos puesto de manifiesto recientemente, "durante los últimos años, se han producido modificaciones en los planteamientos epistemológicos y en la práctica docente de la Educación Mediática (EM) como consecuencia de los cambios producidos por la aparición de nuevas tecnologías y de nuevas prácticas comunicativas" (Ferrés, Masanet y Marta-Lazo, 2013: 130). Especialmente importante se presenta la docencia de esta materia en el ámbito universitario de las titulaciones Educación y Comunicación, ya que se centra en formar a futuros profesionales que ejercerán su labor en esta área específica. Sirvan, como ejemplo, algunas experiencias aisladas en 
las que se introduce al alumno en el proceso de alfabetización audiovisual y lectura crítica de los mensajes de los medios de comunicación, además de acercarlos a los procesos productivos y tecnologías aplicadas a los "media", como es el caso de la Summer Media School (Ortiz Sobrino y Rodríguez Barba, 2011: 4).

\section{OBJETIVOS}

Como ya hemos manifestado, si bien es importante que toda la población posea los niveles mínimos de alfabetización digital o competencia mediática, más necesario es aún para aquellos que se preparan para ser comunicadores y educadores. Por este motivo, en esta investigación abordaremos de qué modo se documentan estos colectivos para conseguir desarrollar esas dimensiones competenciales.

Con esta finalidad, examinaremos todos los recursos didácticos y de aprendizaje que se recomiendan en las guías docentes de las asignaturas directamente relacionadas con la Educación Mediática, tanto en las titulaciones de Comunicación como en las de Educación que se imparten en todo el estado español.

\section{METODOLOGÍA}

El método utilizado para realizar esta investigación ha sido el análisis de contenido de todos los recursos incluidos en la totalidad de guías docentes analizadas durante el curso académico 2012-2013, que sumaron un total de 72 guías docentes (31 en las áreas de comunicación y 41 en las de educación).

Las hipótesis que pretendemos comprobar son las que señalamos a continuación:

- H1: A pesar de la gran cantidad de recursos digitales y audiovisuales existentes, todavía se priorizan los soportes bibliográficos como fuente de aprendizaje.

- H2: La mayoría de recursos recomendados tienen una fecha de edición bastante anticuada, siendo escasas las referencias de los últimos cinco años.

- H3: Las referencias internacionales, prevenientes de otros países, son poco recomendadas en las asignaturas de Educación Mediática, siendo mucho más frecuentes las de ámbito español.

- H4: Los recursos más frecuentes son en lengua española, siendo minoritarios los elaborados en otros idiomas.

\section{RESULTADOS}

De todos los recursos que se recomiendan en las guías docentes de las asignaturas de Educación Mediática, la gran mayoría de los soportes son bibliográficos (libros y capítulos de libro) $(\mathrm{N}=353)$, frente a los hemorográficos $(\mathrm{N}=54)$ y webgráficos $(\mathrm{N}=34)$. En este sentido, comprobamos la hipótesis de partida H1, como puede verse en el Gráfico 1. 
Gráfico 1. Tipología de soportes.

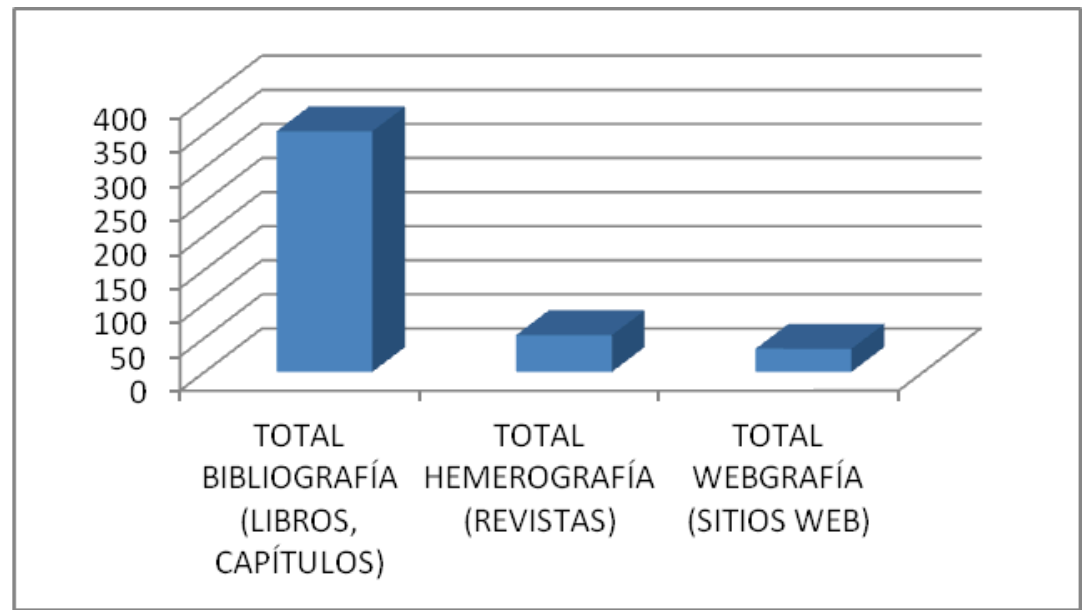

Fuente: Elaboración propia

Respecto a la comparativa entre titulaciones en referencia a los tipos de soporte, se observa una tendencia similar, como puede advertirse en el Gráfico 2, si bien en el caso de las referencias de los estudios de Comunicación el porcentaje de webgrafía es mayor que en los de Educación, suponiendo un $10 \%$ frente a un 4,5\% respecto al total. Sin embargo, sucede lo contrario en el caso de la hemorografía, dado que en los estudios de Comunicación se contiene un 10,4\% de revistas y en los de Educación un $15,7 \%$. Los porcentajes de bibliografía son los más destacados en ambos casos, como ya ha quedado reflejado en el Gráfico anterior.

Gráfico 2. Distribución porcentual de soportes en asignaturas de titulaciones de Educación y de Comunicación.

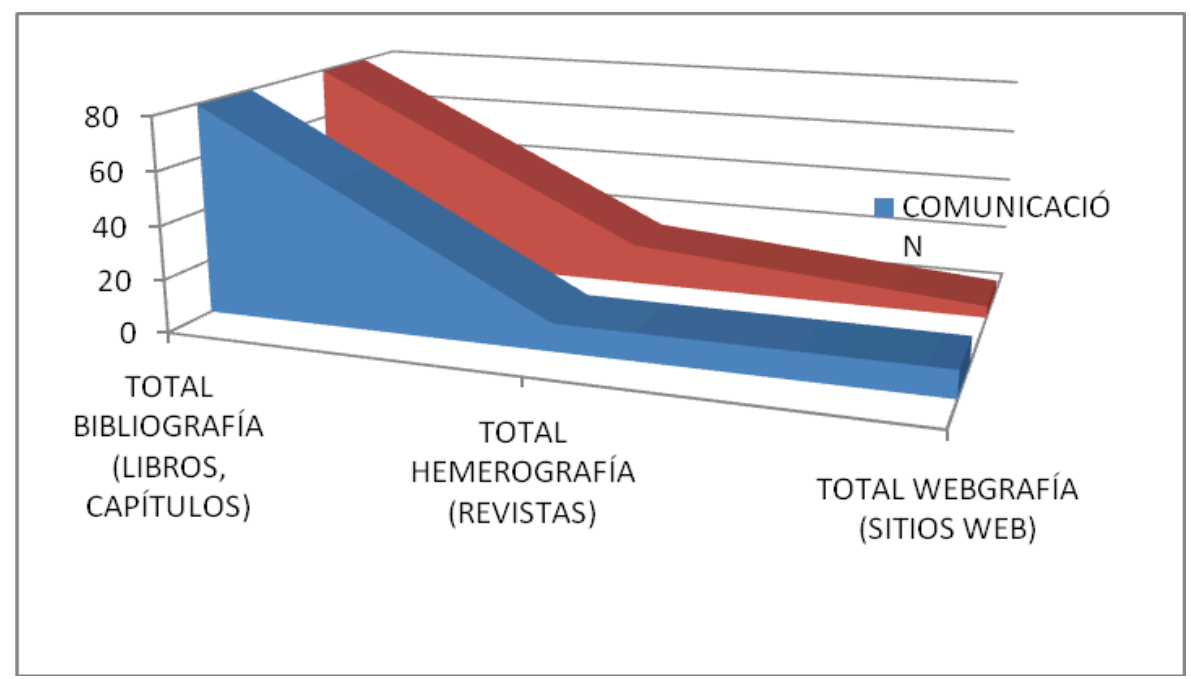

Fuente: Elaboración propia 
Teniendo en cuenta la distribución del total de referencias bibliográficas que se incluyen en el apartado de bibliografía, como puede verse en el Gráfico 3, las más numerosas corresponden a los libros $(\mathrm{N}=351)$, seguidas a gran distancia por artículos $(\mathrm{N}=49)$, capítulos de libro $(\mathrm{N}=26)$, contribuciones de actas de Congresos $(\mathrm{N}=10)$. Cabe destacar que no se incluye ningún DVD entre los materiales recomendados y tan sólo un vídeo.

Gráfico 3. Tipos de referencias en bibliografía.

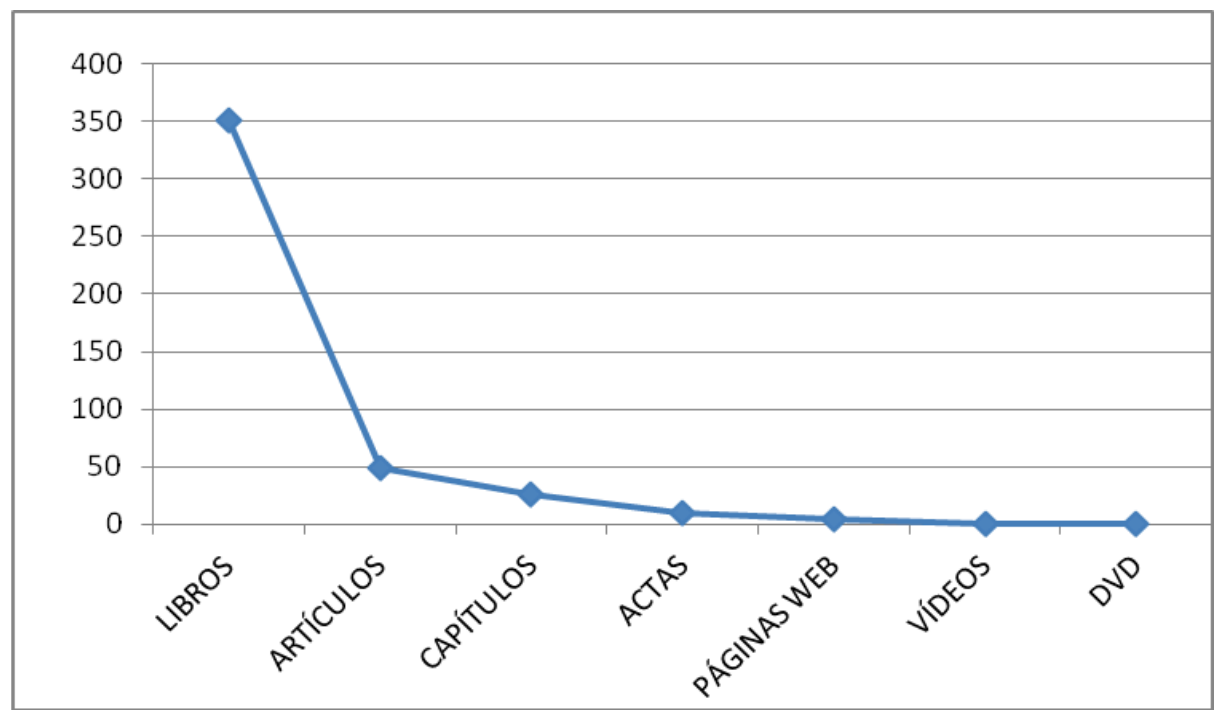

Fuente: Elaboración propia

Cabe destacar como se advierte en el Gráfico 4 que los libros se recomiendan en mayor medida en los estudios de Educación, al igual que los artículos científicos; siendo los capítulos científicos y las contribuciones en Actas de Congresos más frecuentes en las asignaturas de Educación Mediática de las carreras de Comunicación.

Gráfico 4. Reparto tipos de referencias en bibliografía en Educación y Comunicación.

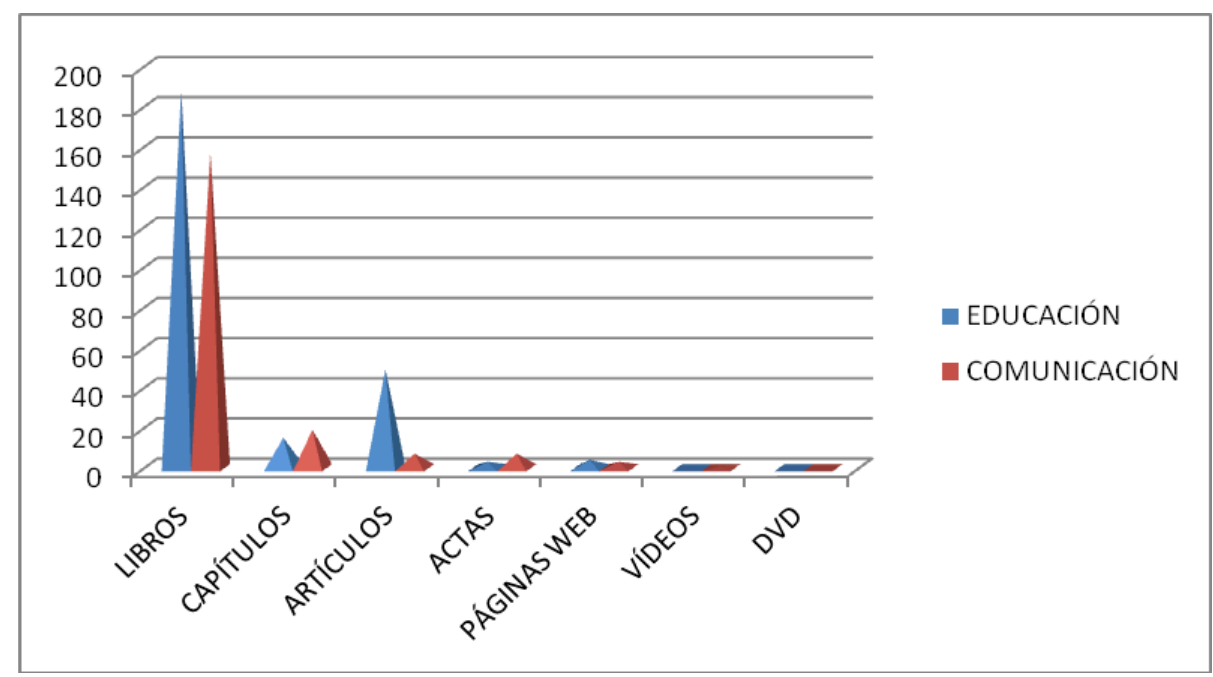

Fuente: Elaboración propia 
En cuanto a la fecha de publicación de los recursos bibliográficos, se confirma la hipótesis 2 (H2), dado que en su mayoría son recursos editados hace más de cinco años. De hecho, el año promedio de publicación de las fuentes incluidas en las guías docentes es el 2001, lo que evidencia que apenas se renuevan los materiales o que no se incluyen los más recientes y se da más importancia a los clásicos o a algunos que han podido quedar anticuados. De esta forma, como puede verse en el Gráfico 5, los más numerosos son los que van de la horquilla de 2000 a 2009 (N=313) y de 1990 a $1999(\mathrm{~N}=169)$.

Gráfico 5. Año publicación de recursos bibliográficos.

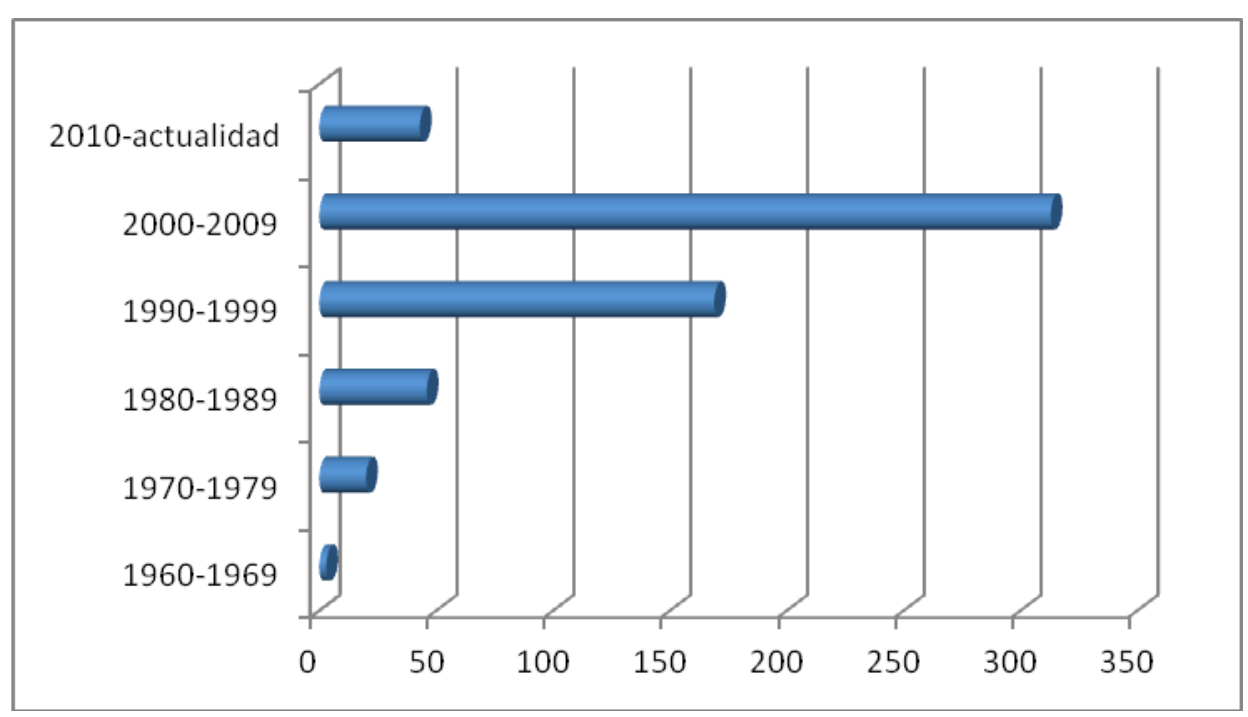

Fuente: Elaboración propia

Atendiendo a la modalidad de bibliografía en la que se integran los recursos, cabe señalar como se aprecia en el Gráfico 6 que en la mayoría de los casos se incluye como bibliografía complementaria $(\mathrm{N}=433)$, aunque también aparece de manera significativa como bibliografía básica ( $\mathrm{N}=406)$. 
Gráfico 6. Modalidad en forma de bibliografía.

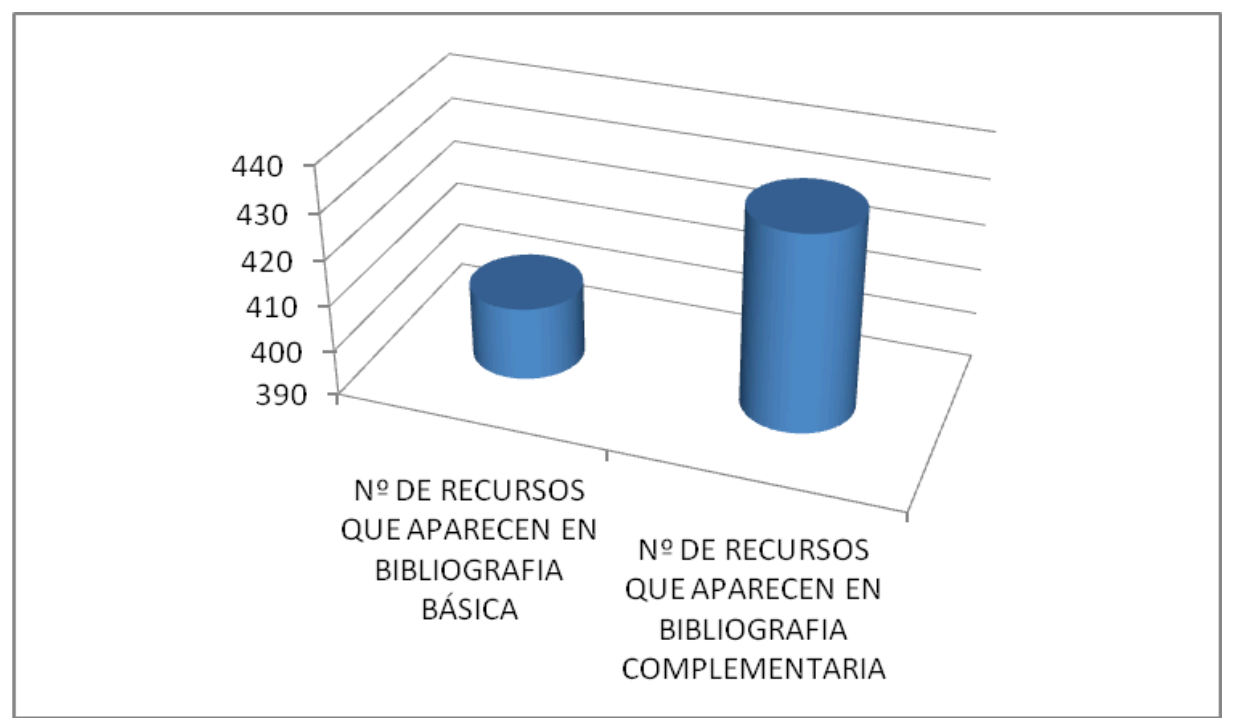

Fuente: Elaboración propia

Es significativa la tendencia contraria en lo que se refiere a modalidad de bibliografía, como se evidencia en el Gráfico 7, siendo más numerosos los recursos de bibliografía básica en las titulaciones de Educación

Gráfico 7. Comparativa distribución bibliografía en asignaturas de educación mediática pertenecientes a titulaciones de Comunicación y de Educación.

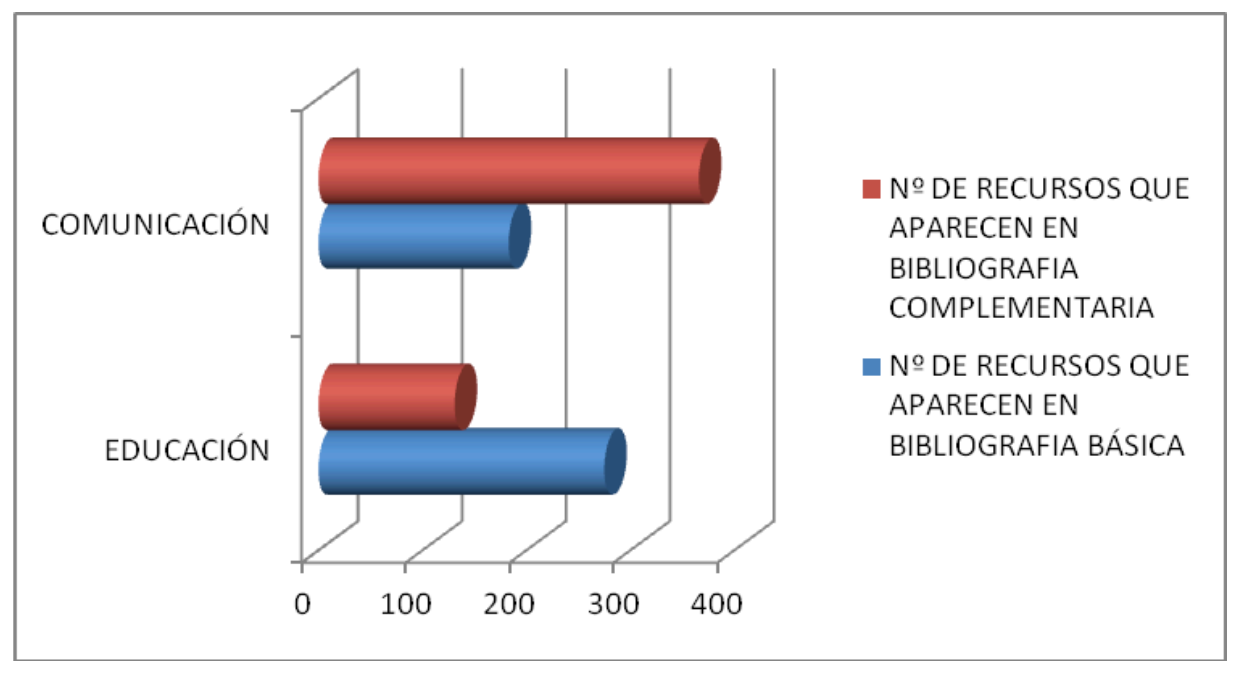

Fuente: Elaboración propia

El origen de los materiales recomendados en las guías docentes de las asignaturas de Educación Mediática también nos hace validar la hipótesis 3 (H3), en la que planteábamos que la mayoría de las referencias son españolas $(\mathrm{N}=632)$, siendo muy escasas las de carácter internacional $(\mathrm{N}=63)$, como se representa en el Gráfico 8. 
Gráfico 8. Procedencia de las referencias bibliográficas.

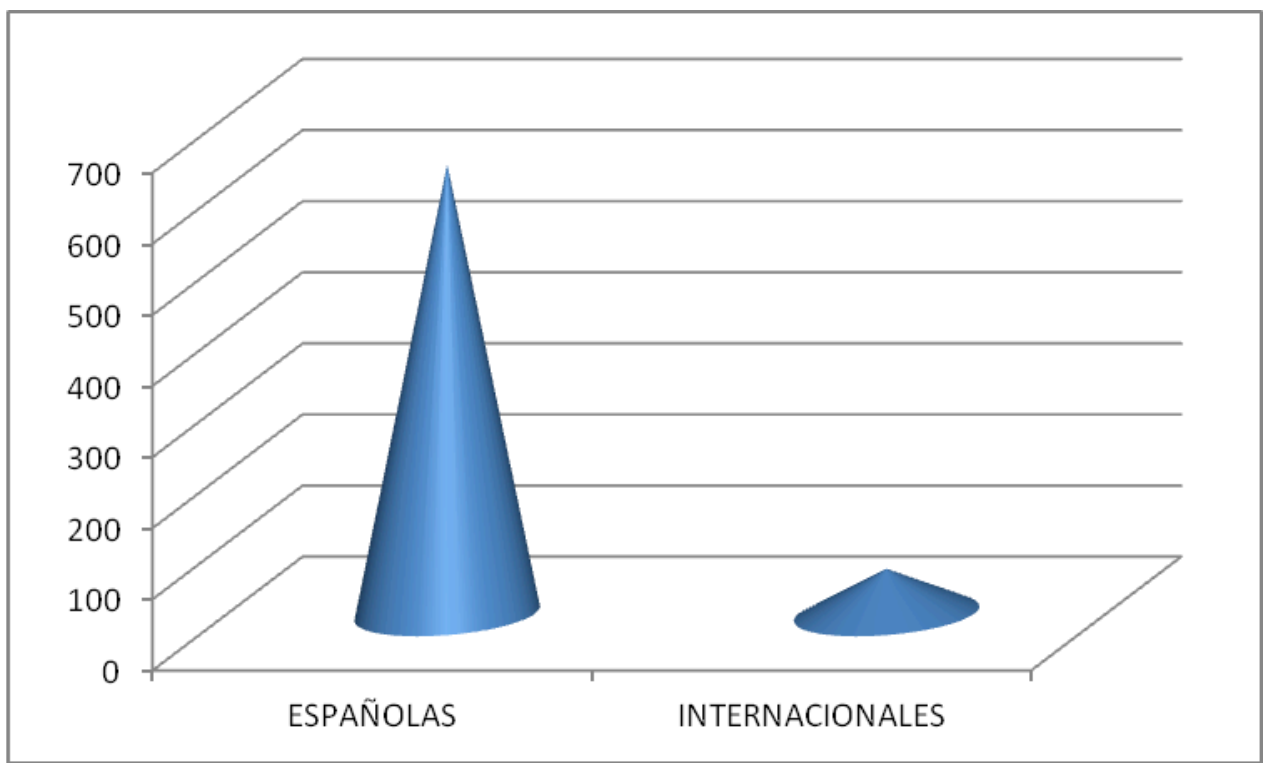

Fuente: Elaboración propia

Siguiendo esta línea, y respecto a los idiomas en los que están elaborados los recursos, como ya avanzábamos en la hipótesis 4 (H4), en su mayoría son en castellano ( $\mathrm{N}=627)$, seguidos del inglés $(\mathrm{N}=57)$, francés $(\mathrm{N}=5)$ y catalán $(\mathrm{N}=5)$, como se puede ver en el Gráfico 9.

Gráfico 9. Idiomas de las referencias bibliográficas.

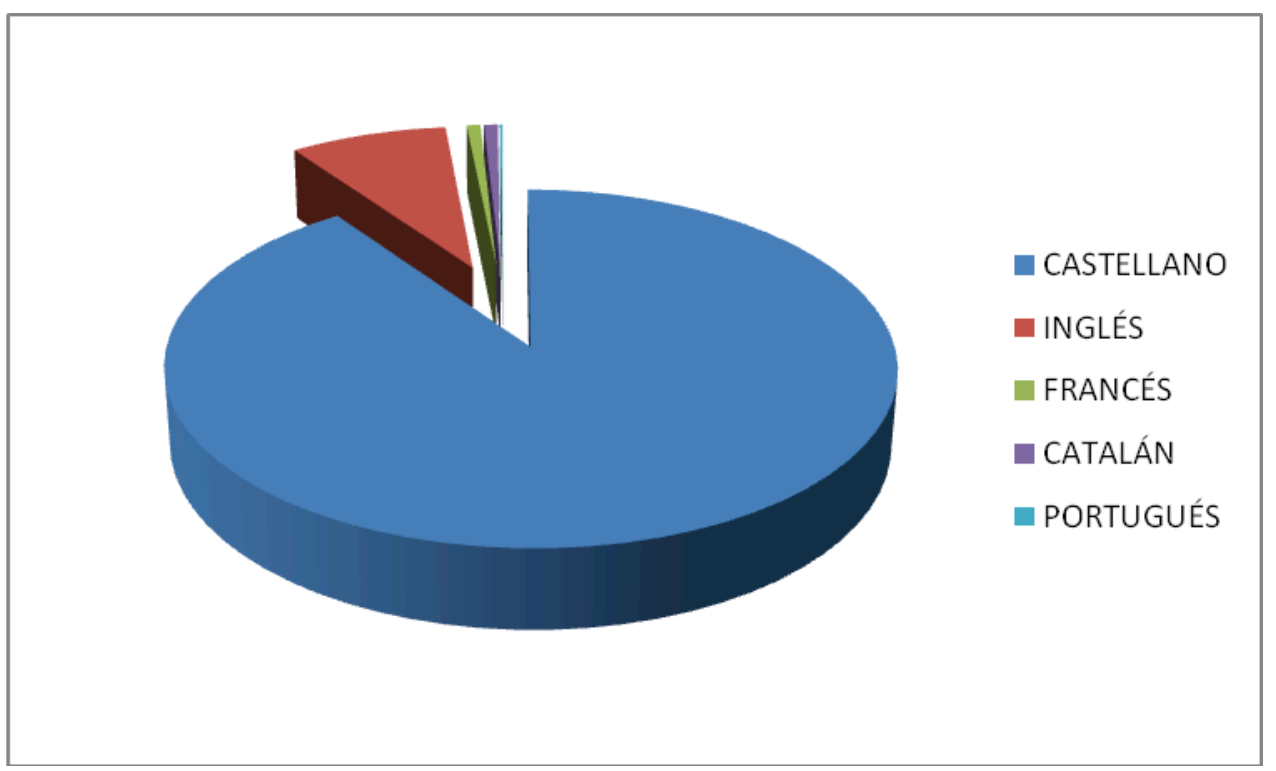

Fuente: Elaboración propia

Teniendo en consideración la escasa tendencia de referenciar recursos internacionales, en el Gráfico 14, puede también comprobarse como la mayoría de los materiales se han 
realizado en España $(\mathrm{N}=632)$ y son muy escasos los originarios de otros países, entre los que siguen Estados Unidos $(\mathrm{N}=54)$ y Francia $(\mathrm{N}=5)$

Gráfico 10. Países de publicación de los recursos recomendados.

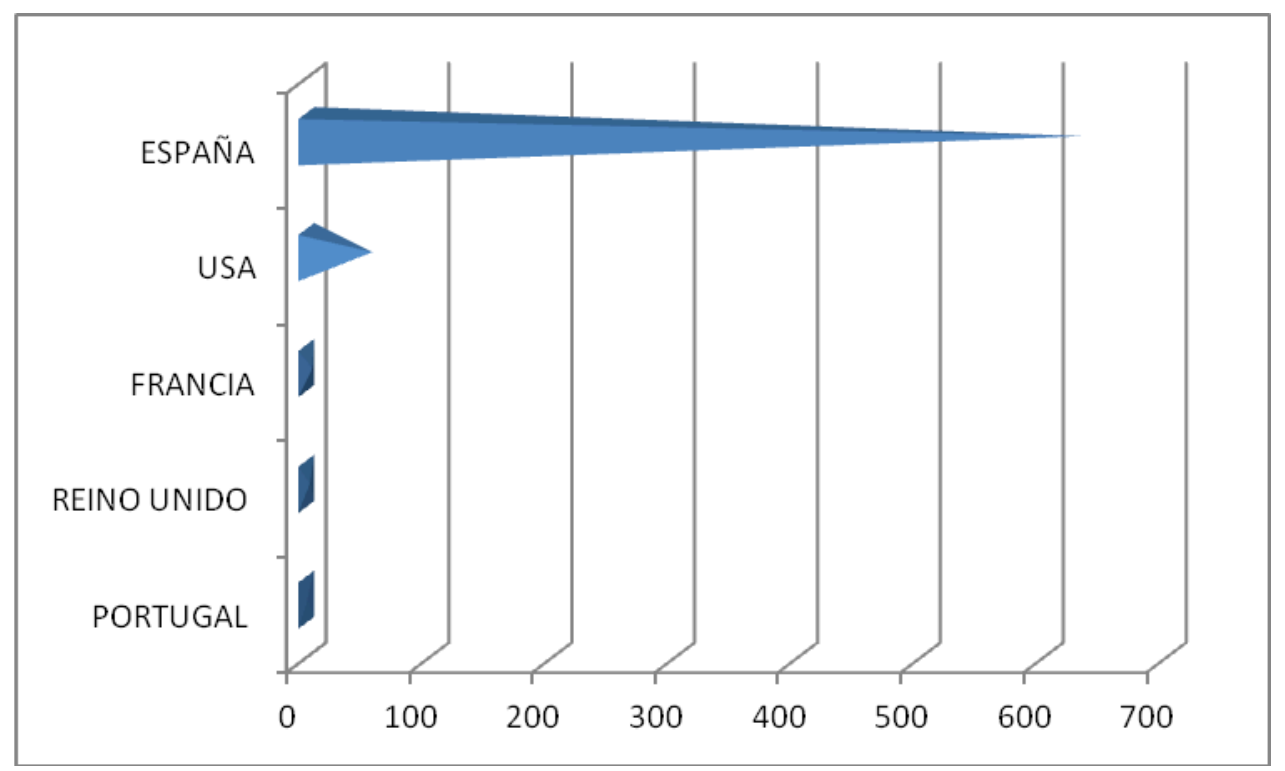

Fuente: Elaboración propia

Por último, en la Tabla 1, indicaremos cuáles son los diez recursos que se referencian un mayor número de veces, correspondiendo todos ellos a libros, como ya era previsible atendiendo a los resultados que hemos mostrado.

Si bien los resultados de los manuales de uso más frecuente serán objeto de otro artículo, simplemente queremos dejar constar que en todos los casos cumplen con las características que hemos ya comprobado fruto del análisis de resultados que nos ha llevado a corroborar todas las hipótesis planteadas. En este sentido, los diez recursos son en soporte bibliográfico, anteriores a 2010, publicados en España y en idioma castellano.

Además, la mayoría se incluyen en las guías docentes de las asignaturas de Educación Mediática como bibliografía básica. Cabe destacar que el autor más referenciado es Joan Ferrés, con tres publicaciones de las diez más numerosas: Educar en una cultura del espectáculo (2000), Televisión y educación (1996) y La educación como industria del deseo (2009). 
Educación mediática - Comunicación - Recursos recomendados - Guías Docentes

Tabla 1. Diez recursos más referenciados en las guías de asignaturas de educación mediática en titulaciones de Educación y Comunicación.

\begin{tabular}{|c|c|c|c|c|c|c|}
\hline & AUTOR & TÍTULO & Año & MODALIDAD & $\begin{array}{c}\mathrm{N}^{\mathbf{o}} \\
\text { VECES }\end{array}$ & $\begin{array}{l}\text { TOTAL } \\
\text { VECES } \\
\end{array}$ \\
\hline \multirow[b]{2}{*}{1} & & \multirow{2}{*}{$\begin{array}{l}\text { Educar en una cultura del } \\
\text { espectáculo }\end{array}$} & \multirow[b]{2}{*}{2000} & Bás & 13 & \multirow[b]{2}{*}{18} \\
\hline & FERRÉS, J. & & & Com & 5 & \\
\hline \multirow[b]{2}{*}{2} & \multirow[b]{2}{*}{ FERRÉS, J. } & \multirow[b]{2}{*}{ Televisión y educación } & \multirow[b]{2}{*}{1996} & Bás & 9 & \multirow[b]{2}{*}{15} \\
\hline & & & & Com & 6 & \\
\hline \multirow[b]{2}{*}{3} & & Tecnología educativa: & & Bás & 13 & \\
\hline & CABERO ALMENARA, J. & vídeo & 2007 & Com & 2 & 15 \\
\hline \multirow[b]{2}{*}{4} & \multirow[b]{2}{*}{ GARCÍA MATILLA, A. } & \multirow[b]{2}{*}{ Una televisión para la educación } & \multirow[b]{2}{*}{2003} & Bás & 11 & \multirow[b]{2}{*}{13} \\
\hline & & & & Com & 2 & \\
\hline \multirow[b]{2}{*}{5} & \multirow[b]{2}{*}{ BUCKINGHAM, D. } & \multirow{2}{*}{$\begin{array}{c}\text { Crecer en la era de los medios } \\
\text { electrónicos }\end{array}$} & \multirow[b]{2}{*}{2002} & Bás & 9 & \multirow[b]{2}{*}{12} \\
\hline & & & & Com & 3 & \\
\hline \multirow[b]{2}{*}{6} & \multirow[b]{2}{*}{ AGUADED GOMEZ, J.I. } & \multirow{2}{*}{$\begin{array}{l}\text { Convivir con la televisión. } \\
\text { Familia, educación y recepción } \\
\text { televisiva }\end{array}$} & \multirow[b]{2}{*}{1999} & Bás & 8 & \multirow[b]{2}{*}{10} \\
\hline & & & & Com & 2 & \\
\hline \multirow[b]{2}{*}{7} & \multirow[b]{2}{*}{ FERRÉS, J. } & \multirow{2}{*}{$\begin{array}{c}\text { La educación como industria del } \\
\text { deseo }\end{array}$} & \multirow[b]{2}{*}{2009} & Bás & 8 & \multirow[b]{2}{*}{10} \\
\hline & & & & Com & 2 & \\
\hline \multirow[b]{2}{*}{8} & \multirow[b]{2}{*}{ BUCKINGHAM, D. } & \multirow{2}{*}{$\begin{array}{c}\text { Educación en medios. } \\
\text { Alfabetización, aprendizaje y } \\
\text { cultura contemporánea }\end{array}$} & \multirow[b]{2}{*}{2005} & Bás & 7 & \multirow[b]{2}{*}{9} \\
\hline & & & & Com & 2 & \\
\hline & & Tecnologías de la Información y & & Bás & 7 & \\
\hline 9 & $\begin{array}{l}\text { CEBRIAN DE LA SERNA, } \\
\text { M. }\end{array}$ & Formación de Docentes & 2005 & Com & 2 & 9 \\
\hline & & & & Bás & 8 & \\
\hline 10 & DE PABLOS, J. & Tecnología Educativa & 2009 & Com & 1 & 9 \\
\hline
\end{tabular}

Fuente: Elaboración propia

\section{DISCUSIÓN}

El desarrollo vertiginoso de la comunicación digital no se acompaña desde el sistema educativo con la enseñanza de las competencias necesarias para estar alfabetizado en el dominio del Lenguaje; Tecnología; Procesos de producción y programación; Ideología y valores; Estética; y Recepción y audiencia. 
En el seno de la Universidad, debería de fomentarse el aprendizaje de los diferentes indicadores competenciales y mucho más en los casos de las titulaciones que preparan para ser comunicadores y educadores. No obstante, como ha quedado demostrado en la investigación que hemos presentado, ni tan siquiera en las asignaturas de Educación Mediática se potencian algunos de estos aspectos. Prueba de ello es que apenas se utilizan recursos digitales ni audiovisuales y que el aprendizaje sigue estando basado en la cultura impresa, incluso el impulsado por parte de quienes se entiende que deberían estar más concienciados en este tema. Como apuntan Osuna Acedo, MartaLazo y Aparici Merino (2012), "la universidad debe potenciar la producción digital y adaptarse al nuevo paradigma, a una nueva forma de trabajar, la cual debe conllevar un cambio de hábitos en los docentes y el incentivo en su reciclaje en el uso de tecnologías digitales". En cuanto a la faceta creativa y la preparación hacia el ejercicio profesional, "La convergencia multimedia repercute en que los nuevos comunicadores necesitan conocer todos los códigos (impreso, fotográfico, auditivo, visual, audiovisual, digital). La preparación en el análisis y ejecución de contenidos para cualquier soporte, dota a los graduados de un aprendizaje integral" (Vadillo, MartaLazo y Cabrera, 2010: 186). En este sentido, conviene resaltar la importancia de fomentar la creación de material didáctico para la enseñanza de la educación mediática en España ya que la mayoría de los recursos recomendados son manuales o artículos científicos que profundizan en la naturaleza de esta materia, pero no tanto en su ejecución práctica docente. En este sentido, sería conveniente que dichos materiales didácticos pudieran estar disponibles en Internet con acceso libre para futuros y presentes educomunicadores. Esta creación tiene puerta abierta a un paraíso de gratificaciones que ofrecen estas narrativas, que precisan emprender una pedagogía del conflicto que entienda que el impacto multimedia es básicamente emotivo, contextualmente lúdico y cognitivamente social (Gabelas, 2010: 218).

Como ya se ha puesto de manifiesto, la mayoría de material recomendado por las universidades españolas es material científico: o bien académico o bien artículos provenientes de proyectos de investigación. Esto demuestra la vitalidad y esfuerzos exponenciales que desde los diferentes grupos de investigación de la universidad española se están realizando para profundizar en el ámbito de la educomunicación. Sería recomendable para los profesores encargados de materias relacionadas con esta área de conocimiento que actualizaran su repertorio con las últimas investigaciones que se están desarrollando recientemente. La mayoría de recursos referenciados en las asignaturas de Educación Mediática son bastante antiguos, lo que evidencia dos aspectos: la falta de reciclaje y también la frecuencia con la que se incluyen fuentes clásicas, como se pone de manifiesto en casi todos los recursos que mayor número de veces se recogen en la bibliografía. Los diez más citados se corresponden con los grandes autores de la Educación Mediática en el ámbito español, tales como Joan Ferrés, Julio Cabero, Agustín García Matilla, David Buckingham, José Ignacio Aguaded, Manuel Cebrián de la Serna y Juan de Pablos.

Respecto a la recomendación de autores de referencia, es llamativo cómo a pesar de estar ya totalmente implantado el Espacio Europeo de Educación Superior (Caldevilla, 2012), en las asignaturas de Educación Mediática apenas se utilizan recursos internacionales ni en otros idiomas diferentes al castellano. Apenas se referencian 
materiales en inglés ni autores provenientes de otros contextos culturales en los que se desarrollan aspectos fundamentales de la media literacy. El único autor extranjero en los 10 manuales más recomendados es David Buckingham y en la traducción al español de dos de sus obras. Sin embargo, trabajos importantes como los desarrollados por Sonia Livingstone o Divina Frau-Meigs en el contexto europeo, apenas están referenciados en el análisis de las 72 guías docentes. Conviene destacar también en estas líneas los trabajos realizados por el grupo de trabajo sobre media literacy liderado por Sonia Livingstone en la Acción Cost "Transforming Audiences, Transforming Societies" (2010-2014), que durante 4 años han coordinado esfuerzos para valorar el presente y futuro inminente de la enseñanza de la educación en medios en Europa (Livingstone et al, 2012; Livingstone et al, 2014) y que podría ser de interés para futuros educomunicadores. En esta misma línea, sorprende la ausencia total de referencias al estudio y aplicación de los videojuegos, que en autores como Ito, Rheingold o Jansz.

Del mismo modo, cabe señalar que tampoco encontramos entre los diez recursos más frecuentes referencias de ningún autor latinoamericano, siendo que la corriente de estudios de Educomunicación se fragua y afianza con potencialidad en aquellos países en los que se fomenta desde la educación para el desarrollo. En este sentido, también se echan en falta autores como Kaplún, Orozco, Prieto, Fuenzalida, Hermosilla o Quiroz, entre otros.

\section{REFERENCIAS}

AGUADED, J. I. (2011): "Media education: An internacional unstoppable phenomenon. The Work of the UN, Europe and Spain in the Field of Educommunication", Comunicar, $\mathrm{n}^{\mathrm{o}} 37$, vol. 19, p. 7-8.

AA.VV. (1982): “Declaración sobre la enseñanza de los medios, Actas Simposium Internacional sobre la Enseñanza de los Medios". Grunwal, Alemania. Recuperado el 21.07.2013

de: http://mediascopio.org/Documentacion\%20Mediascopio/Documentac_Unesco_ense nanza_medios.htm

CALDEVILLA DOMÍNGUEZ, D. (Coord.) (2012): El reto en la innovación docente: el EEES como punto de encuentro. Madrid: Visión Libros.

COMMUNICATION FROM THE COMMISSION TO THE EUROPEAN PARLIAMENT, THE COUNCIL, THE EUROPEAN ECONOMIC AND SOCIAL COMMITTEE AND THE COMMITTEE OF THE REGIONS (2007): “A European approach to media literacy in the digital environment", Brussels, COM, 833 final, pp. $1-9$.

FERRÉS PRATS, J.; MASANET JORDÁ, M.-J. y MARTA-LAZO, C. (2013): Historia y Comunicación Social, Vol. 18. No Esp. Dic., pp. 129-144. 
FERRÉS, Joan, GARCÍA MATILLA, Agustín, AGUADED, Juan Ignacio; FERNÁNDEZ CAVIA, Josep, FIGUERES, Mónica, BLANES, Magda et al. (2011): Competencia mediática. Investigación sobre el grado de competencia de la ciudadanía en España. Madrid: Gobierno de España. Ministerio de Educación, Instituto de Tecnologías Educativas y Formación del Profesorado.

FRAU-MEIGS, D. (2012): “Transliteracy as the new research horizon for media and information literacy", Media Studies, Vol 3 (6), pp. 14-27.

GABELAS BARROSO, J.A (2007): “Una perspectiva de la educación en medios para la comunicación en España". En Revista Comunicar, La educación en medios en Europa. ${ }^{\circ}$ 28, pp. 69-73.

GABELAS BARROSO, J. A. y MARTA-LAZO, C. (2011): "Adolescentes en la cultura digital". En MARTÍNEZ RODRIGO, E. y MARTA-LAZO, C. (coord..): Jóvenes interactivos, nuevos modos de comunicarse. Netbiblo, pp. 3-15.

GABELAS BARROSO, J. A. (2010): "Escenarios virtuales, cultura juvenil y educomunicación 2.0. " En Educomunicación: más allá del 2.0. Gedisa, pp. 205-223.

LIVINGSTONE, S., WIJNEN, C.; PAPAIOANNOU, T., COSTA, C. GRANDÍO, M. (2014): "Situating media literacy in the changing media environment: critical insights from European Research on Audiences". En CARPENTIER, N.; SCHRODER, K.; HALLET, L. Audiences Transformations. Shifting Audiences Positions in Later Modernity, Routledge, New York and London, pp. 210-227.

LIVINGSTONE, S., PAPAIOANNOU, T., GRANDÍO PEREZ, M., WIJNEN, C. (2012): "Critical insights in European Media Literacy Research and Policy", Media Studies, Vol $3(6)$, pp. 2-13.

MARTA-LAZO, C. y GRANDÍO PÉREZ, M. (2013): “Análisis de la competencia audiovisual de la ciudadanía española en la dimensión de recepción y audiencia", CommunicationESociety/Comunicación y Sociedad, Vol. 26, n. 2, pp. 114-130.

MARTA-LAZO, C. y GRANDÍO PÉREZ, M. (2012): “Critical insights in media literacy research in Spain: educational and political challenges, Vol. 3, (6), pp. 139-151. 
ORTIZ SOBRINO, M. Á. y RODRÍGUEZ BARBA, D. (2011): “El perfil de entrada de los alumnos de grado en las facultades de comunicación de las universidades de Madrid: el caso de la Summer Media School de la fundación FIDES/UFV". En Vivat Academia. $\quad \mathrm{n}^{\mathrm{o}} \quad 114 . \quad$ Recuperado el 15.12 .2013 de: www.ucm.es/info/vivataca/numeros/n114/DATOSS.htm

OSUNA ACEDO, S.; MARTA-LAZO, C. y APARICI MERINO, R. (2013): “Valores de la formación universitaria de los comunicadores en la Sociedad Digital: más allá del aprendizaje tecnológico, hacia un modelo educomunicativo", Razón y Palabra, № 81.

VADILLO BENGOA, N.; MARTA-LAZO, C.; y CABRERA ALTIERI, D. (2010): "Proceso de adaptación de los estudios de Comunicación al EEES. El caso de Aragón, una comunidad pionera", Revista Latina de Comunicación Social, No 65, pp. 187- 203.

\section{Carmen Marta-Lazo}

Profesora Titular de Comunicación Audiovisual y Publicidad de la Universidad de Zaragoza. Doctora en Ciencias de la Información por la Universidad Complutense de Madrid. Investigadora Principal del Grupo de Investigación en Comunicación e Información Digital (GICID). Responsable de Política universitaria y Foros científicos del Observatorio del Ocio y del Entretenimiento Digital (OCENDI). Miembro del proyecto I+D del Ministerio de Ciencia e Innovación "La enseñanza universitaria ante la competencia en comunicación audiovisual en un entorno digital" (2011-2013). Es Secretaria de Redacción de la Revista Mediterránea de Comunicación y miembro del Comité Científico de Revista Latina, Enl@ce, Icono 14, ZER, @tic, Ámbitos, Pangea, Novarum, entre otras. Ha escrito más de cincuenta publicaciones entre libros, capítulos y artículos relacionadas con la Educación Mediática.

\section{María del Mar Grandío}

Doctora por la Universidad de Navarra y profesora de Ficción Seriada Audiovisual en la Universidad de Murcia. Colabora con la UNIR en el Máster Universitario en Creación de Guiones Audiovisuales. En la actualidad, centra su investigación en competencias mediáticas en un entorno digital, consumo a través de nuevos soportes audiovisuales, audiencias crossmedia y fenómeno fan. Es miembro del Comité de Gestión de la Acción Cost, "Transforming Audiences, Transforming Societies" (20102014) financiado por la Unión Europea y participa en el proyecto de investigación financiado por el Ministerio de Ciencia e Innovación "La enseñanza universitaria ante la competencia en comunicación audiovisual en un entorno digital" (2011-2013). 


\section{José Antonio Gabelas Barroso}

Profesor de Comunicación Audiovisual y Publicidad de la Universidad de Zaragoza. Doctor en Ciencias de la Información por la Universidad Complutense de Madrid. Licenciado en Historia del Arte por la Universidad de Zaragoza. Diplomado en Cinematografía por la Cátedra de Cine de Valladolid. Diplomado en E.G.B por la Universidad de Zaragoza. Diplomado en Animación Socio-Cultural por la Universidad Pontificia de Salamanca. Ha sido director 1998-2006 de la Colección Comunicación y Medios. Ha escrito en múltiples publicaciones capítulos y artículos relacionados con la Educación Mediática. 